\title{
Spin contribution to the ponderomotive force in a plasma
}

\author{
G. Brodin, A. P. Misra, and M. Marklund* \\ Department of Physics, Umeå University, SE-901 87 Umeå, Sweden
}

(Dated: 24 March, 2010; Revised 27 July, 2010)

\begin{abstract}
The concept of a ponderomotive force due to the intrinsic spin of electrons is developed. An expression containing both the classical as well as the spin-induced ponderomotive force is derived. The results are used to demonstrate that an electromagnetic pulse can induce a spin-polarized plasma. Furthermore, it is shown that for certain parameters, the nonlinear back-reaction on the electromagnetic pulse from the spin magnetization current can be larger than that from the classical free current. Suitable parameter values for a direct test of this effect are presented.

PACS numbers: 52.35.Mw, 52.27.-h, 52.38.-r
\end{abstract}

The use of the spin properties of material constituents for e.g., carrying information is currently an important paradigm [1]. However, the spin properties of the material constituents also make its presence felt through collective effects. In particular, recent findings point to the possibility of observing quantum plasma effects [2] through the electron spin [3] in regimes otherwise thought to be classical [4]. Such results are due to the complex interplay between collective plasma effects and the system nonlinearities. In classical plasmas, nonlinear effects play an important, sometimes a crucial, role. For example, the density fluctuations induced by the ponderomotive force of an electromagnetic (EM) wave lead to an electrostatic wake field [5], as used in advanced particle accelerator schemes [6]. In other regimes, the back-reaction on the EM-wave due to the density fluctuations leads to phenomena such as soliton formation, self-focusing or wave collapse [7]. Such radiation pressure-like effects are widely used in high-intensity laser experiments [8], and generalizations to include certain types of quantum plasma effects have recently been made [9]. However, to our knowledge the possibility of spin induced contribution to the ponderomotive forces has not been explored.

In the present work we will solve the full set of equations for the spin dynamics of charged particles in the presence of a weakly nonlinear EM wave pulse, propagating parallel to an external magnetic field, in order to find the contribution to the ponderomotive force. In the classical limit, we recover the well-known expression first derived by Karpman and Washimi [14]. The spin contribution to the ponderomotive force will in general act in opposite directions for spin-up and spin-down populations. As a consequence, an EM-pulse (due to, e.g., a laser or a microwave source) may induce a spin-polarized plasma. In particular, it is demonstrated that this mechanism can induce large spin-polarization for a laser source in the UV-regime. For this case it should be noted that the effect of the external magnetic field is negligible as the laser frequency is much higher than the cyclotron frequency, but our general expression applies also for lowfrequency (lf) waves in magnetized plasmas.

When combined with the high-frequency (hf) oscilla- tions, the classical lf density response generates a hf current that results in a cubical nonlinearity. This classical nonlinearity can be compared to a cubically nonlinear magnetization current, caused by the electrons spins in a plasma that are spin-polarized due to ponderomotive effects. It turns out that for a plasma frequency corresponding to a metal density and a hf source (e.g., an x-ray free electron laser (XFEL)), the spin contribution can be larger than the classical contribution.

We will assume the existence of two electron populations, namely spin-up and spin-down relative to a background magnetic field $\mathbf{B}_{0} \equiv B_{0} \hat{\mathbf{z}}$, to be denoted by $u$ and $d$ respectively, and formally treated as different species. Such division are relatively common in e.g. semiconductor physics [10]. Although the spin states of the particles will be perturbed by the presence of electromagnetic waves, the separation of species is still well-defined provided the physics associated with spin-flips can be neglected (see Refs. [4] and [11] for a discussion and a calculation of spin-flip frequencies, respectively). The basic equations take the form $[\underline{3}, 4]$

$$
\begin{aligned}
\partial_{t} n_{\alpha}+\nabla \cdot\left(n_{\alpha} \mathbf{v}_{\alpha}\right) & =0, \\
m\left(\partial_{t}+\mathbf{v}_{\alpha} \cdot \nabla\right) \mathbf{v}_{\alpha} & =q\left(\mathbf{E}+\mathbf{v}_{\alpha} \times \mathbf{B}\right)-\nabla P_{\alpha} / n_{\alpha} \\
& +(2 \mu / \hbar) S_{\alpha}^{a} \nabla B_{a}, \\
\left(\partial_{t}+\mathbf{v}_{\alpha} \cdot \nabla\right) \mathbf{S}_{\alpha}= & -(2 \mu / \hbar) \mathbf{B} \times \mathbf{S}_{\alpha},
\end{aligned}
$$

and $\nabla \cdot \mathbf{E}=\left(q / \varepsilon_{0}\right)\left(n_{u}+n_{d}-n_{i}\right)$, where $\mathbf{S}_{\alpha}$ is the spin of species $\alpha$ (with $\alpha=u, d$ ), $n_{i}$ is the ion density, and $q=-e$ is the electron charge, $\mu \equiv-g \mu_{B} / 2$, where $\mu_{B} \equiv e \hbar / 2 m$ is the Bohr magneton, $g \approx 2.0023192$ is the electron $g$-factor, and $P_{\alpha}$ is the pressure [12]. The principal condition for the validity of Eqs. (1)-(3) is that characteristic spatial scale lengths is longer than the thermal de Broglie length. Naturally Eqs. (11)-(3) also has the inherent limitations of a fluid model, i.e. resonance effects for particle velocities matching the phase velocity and/or group velocity are left out. Furthermore, effects due to the off-diagonal componenets of the pressure tensor are omitted. In what follows we consider an electron-proton plasma with classical ion dynamics.

Our derivation of the ponderomotive force below will 
be based on a direct perturbative analysis of Eqs. (1)(3). We note that an alternative approach based on Lagrangian method [15, 16] can be useful. Assuming a slowly varying plane $\mathrm{EM}$ wave $\mathbf{E}=\widetilde{\mathbf{E}} \exp [i(k z-\omega t)]+$ c.c., where c.c. denotes complex conjugate, Eq. (2) becomes

$$
\left(\partial_{t}-i \omega\right) \mathbf{v}_{\alpha}=(q / m) \mathbf{E}-\Omega \mathbf{v}_{\alpha} \times \hat{\mathbf{z}}
$$

where $\Omega \equiv e B_{0} / m$ is the electron-cyclotron frequency. Here we have assumed that the background state contains no net drifts. Note that kinetic effects, which is of potential importance (see e.g. [17]) but is outside the present model, is assumed to be negligible throughout the manuscript. Within a fluid model, the average longitudinal velocity, that may contribute through the convective derivative (cf. Ref. [15]) becomes second order in the amplitude, and hence can be neglected here. For notational convenience, we have dropped the tilde denoting the envelope function, and it is understood that all derivatives act on the slowly varying amplitudes. Next, we define the variables $v_{\alpha \pm} \equiv v_{\alpha x} \pm i v_{\alpha y}, E_{ \pm} \equiv E_{x} \pm i E_{y}$. Substituting the lowest order result $v_{\alpha \pm}=i q E_{ \pm} / m(\omega \pm \Omega)$ into the correction term in Eq. (4), we obtain

$$
v_{ \pm} \equiv v_{\alpha \pm}=\frac{q}{m} \frac{1}{(\omega \pm \Omega)}\left[i E_{ \pm}+\frac{1}{\omega \pm \Omega} \frac{\partial E_{ \pm}}{\partial t}\right]
$$

Using Faraday's law, $\nabla \times \mathbf{E}=-\partial_{t} \mathbf{B}$, we similarly obtain the expression for the perturbed magnetic field as

$$
B_{ \pm}= \pm \frac{i k}{\omega} E_{ \pm} \pm \frac{1}{\omega} \frac{\partial E_{ \pm}}{\partial z} \pm \frac{k}{\omega^{2}} \frac{\partial E_{ \pm}}{\partial t} .
$$

The classical ponderomotive force component is

$$
F_{\mathrm{c} z} \equiv \frac{q}{m}\langle\mathbf{v} \times \mathbf{B}\rangle_{z}=\left\{\begin{array}{l}
\frac{i q}{2 m}\left(v_{+} B_{+}^{*}-v_{+}^{*} B_{+}\right) \text {for RCP } \\
\frac{i q}{2 m}\left(v_{-}^{*} B_{-}-v_{-} B_{-}^{*}\right) \text { for LCP }
\end{array}\right.
$$

Substitution of Eqs. (5) and (6) into Eq. (17) gives

$$
F_{\mathrm{c} z}=-\frac{e^{2}}{2 m^{2} \omega(\omega \pm \Omega)}\left[\frac{\partial}{\partial z} \pm \frac{k \Omega}{\omega(\omega \pm \Omega)} \frac{\partial}{\partial t}\right]|E|^{2}
$$

in agreement with the classical result [14].

Next, we derive the effects due to the finite magnetic moment of the electrons. Through the force $F_{\alpha z} \equiv$ $(2 \mu / m \hbar)\left\langle S_{\alpha}^{a} \nabla B_{a}\right\rangle_{z}$ in the averaged momentum equation, a ponderomotive effect due to spin will be generated, where the EM wave will lead to the separation of the spin-up and down electrons, as will be shown below. Starting from the linearized spin-evolution equation

$$
\left(\partial_{t}-i \omega\right) \mathbf{S}_{\alpha}=-(2 \mu / \hbar)\left(B_{0} \hat{\mathbf{z}} \times \mathbf{S}_{\alpha}+S_{0 \alpha} \mathbf{B} \times \hat{\mathbf{z}}\right),
$$

where $S_{0 u}=\hbar / 2=-S_{0 d}$, the contribution from the magnetic dipole force can be obtained. Neglecting the slow time derivative, Eq. (9) gives

$$
S_{\alpha \pm} \equiv S_{\alpha x} \pm i S_{\alpha y}=\mp 2 \mu S_{0 \alpha} B_{ \pm} /\left[\hbar\left(\omega \pm \omega_{g}\right)\right]
$$

where $\omega_{g} \equiv g \mu_{B} B_{0} / \hbar=(g / 2) \Omega$ is the spin-precession frequency. Then, including the first order correction, the expression for the perturbed spin becomes

$$
S_{\alpha \pm}=\frac{2 \mu S_{0 \alpha}}{\hbar\left(\omega \pm \omega_{g}\right)}\left[\mp B_{ \pm} \pm \frac{i}{\left(\omega \pm \omega_{g}\right)} \frac{\partial B_{ \pm}}{\partial t}\right] .
$$

The spin-ponderomotive force can be written as

$$
F_{\alpha z}=(2 \mu / m \hbar)\left(S_{\alpha \pm} \partial_{z} B_{ \pm}^{*}+S_{\alpha \pm}^{*} \partial_{z} B_{ \pm}\right) .
$$

Substitution of Eq. (11) into Eq. (12) gives

$$
F_{\alpha z}=\mp \frac{4 \mu^{2}}{m \hbar^{2}} \frac{S_{0 \alpha}}{\left(\omega \pm \omega_{g}\right)}\left[\frac{\partial}{\partial z}-\frac{k}{\left(\omega \pm \omega_{g}\right)} \frac{\partial}{\partial t}\right]|B|^{2} .
$$

The above expression applies to arbitrary EM wave propagation parallel to $\mathbf{B}_{0}$. The overall structure of the spin ponderomotive part of the force (13) is similar to the classical part (8). However, there are differences. Firstly, the frequency resonances occur at the spin precession frequency $\omega_{g}=(g / 2) \Omega \approx 1.00116 \Omega$. Secondly, the dependence on the unperturbed spin state means that spin-up and spin-down populations are pushed in opposite directions by the spin force. Thirdly, for frequencies well below the cyclotron frequency typically the part of the spin contribution proportional to the time-derivative is negligible, whereas it is crucial for the classical contribution. For frequencies well above the cyclotron frequency, the force ratio scaling is $\left|F_{\alpha z}\right| /\left|F_{\mathrm{c} z}\right| \equiv \hbar k\left(1+v_{g} / v_{p}\right) / m v_{p} \sim$ $\hbar k / m c$ for $v_{g}, v_{p} \sim c$, where $v_{g(p)}$ is the group (phase) speed of the wave. As we will see below, even a rather weak spin-ponderomotive force, corresponding to moderately high frequencies, can lead to large modifications of the nonlinear dynamics in an unmagnetized plasma.

We now use the expressions for the ponderomotive forces as source terms for longitudinal if perturbations. We define $N_{1,2}=n_{u} \pm n_{d}$ and $V_{1,2}=\left(v_{u} \pm v_{d}\right) / 2$. In what follows, we will also neglect any difference in the unperturbed spin populations, i.e., we will use $n_{0 u}=$ $n_{0 d} \equiv n_{0} / 2$, which is a good approximation when the Zeeman energy is smaller than the thermal energy. From the lf parts of the continuity equations for spin-up $(u)$ and spin-down $(d)$ populations we then have

$$
\partial_{t} N_{1,2}=-n_{0} \partial_{z} V_{1,2}
$$

From the momentum balance equations, using Eq. (6), we obtain for the lf response the equations

$$
\frac{\partial V_{1}}{\partial t}=\frac{q}{m} E_{l}-\frac{q^{2}}{2 m^{2} \omega(\omega \pm \Omega)}\left[\frac{\partial}{\partial z} \pm \frac{k \Omega}{\omega(\omega \pm \Omega)} \frac{\partial}{\partial t}\right]|E|^{2},
$$

and

$$
\frac{\partial V_{2}}{\partial t}=\mp \frac{4 \mu^{2} k^{2} S_{0}}{m \hbar^{2} \omega^{2}\left(\omega \pm \omega_{g}\right)}\left[\frac{\partial}{\partial z}-\frac{k}{\left(\omega \pm \omega_{g}\right)} \frac{\partial}{\partial t}\right]|E|^{2},
$$


where $E_{l}$ is the lf part of the electric field, $S_{0}=\hbar / 2$ and $\omega_{p}=\left(n_{0} q^{2} / m \varepsilon_{0}\right)^{1 / 2}$. Here we have neglected thermal effects, which is justified if $v_{\text {th }}^{2} \ll v_{g}^{2}$, where $v_{\text {th }}$ is the thermal velocity. Similarly particle dispersive effects and Fermi pressure effects, which may influence the longitudinal dynamics [18], has been neglected, which can be justified in the example considered below [19]. With immobile positive charge carriers, Poisson's equation is $\partial_{z} E_{l}=\left(q / \varepsilon_{0}\right)\left(N_{1}-n_{0}\right)$. Together with Eqs. (14)-(15), we then obtain the wave equations

$v_{g}^{2} \frac{\partial^{2} N_{1}}{\partial \xi^{2}}+\omega_{p}^{2} N_{1}=\frac{\varepsilon_{0} \omega_{p}^{2}}{2 m \omega(\omega \pm \Omega)}\left[1 \mp \frac{k v_{g} \Omega}{\omega(\omega \pm \Omega)}\right] \frac{\partial^{2}|E|^{2}}{\partial \xi^{2}}$,

$$
v_{g}^{2} \frac{\partial^{2} N_{2}}{\partial \xi^{2}}= \pm \frac{\varepsilon_{0} \omega_{p}^{2} k^{2} S_{0}}{2 m^{2} \omega^{2}\left(\omega \pm \omega_{g}\right)}\left[1+\frac{k v_{g}}{\left(\omega \pm \omega_{g}\right)}\right] \frac{\partial^{2}|E|^{2}}{\partial \xi^{2}},
$$

where we have transformed to a comoving frame, with $\xi=z-v_{g} t$. The spin polarization [see Eq. (17)], can be integrated directly to give $N_{2} \propto|E|^{2}$, whereas $N_{1}$ is non-locally related to $|E|^{2}$ through (16), due to the possible excitation of a plasma oscillation wakefield with a characteristic wavelength $\lambda_{p} \equiv v_{g} / \omega_{p}$. To demonstrate that the spin effects can be significant also when $B_{0} \rightarrow 0$, we compare the amplitude of the total density perturbation $N_{1}$ with the degree of spin-polarization $N_{2}$ in an unmagnetized plasma $\omega_{g}, \Omega \rightarrow 0$. Furthermore, to be specific, we consider hf EM waves with $v_{g}, v_{p} \sim c$. Finally, we use $\omega_{p} \lesssim k c$ and use the estimate $\partial^{2}|E|^{2} / \partial \xi^{2} \sim|E|^{2} / L_{p}^{2}$, where $L_{p} \gg k^{-1}$ is the length of the hf pulse. The degree of spin-polarization is then $N_{2} / N_{1} \sim \hbar \omega_{p}\left(k L_{p}\right)^{2} / m c^{2}$. We note that the omission of ion density dynamics constrains this expression to pulse lengths fulfilling $L_{p} \lesssim c / \omega_{p i}$, where $\omega_{p i}$ is the ion plasma frequency. Thus, here we will consider the case of an EM-pulse interacting with a plasma without positive mobile charge carriers, i.e., a metal with $\omega_{p} / 2 \pi \simeq 10^{16} \mathrm{~s}^{-1}$. A numerical example with a UV-laser of wavelength, $\lambda=80 \mathrm{~nm}$ and pulse length, $L_{p}=15 \mu \mathrm{m}$ leading to moderate spin-polarization $\left(N_{2} / N_{1} \approx 3\right.$ at the centre) is displayed in Fig. 1. A longer pulse length or a shorter wavelength will give a higher degree of spinpolarization, i.e. a strongly spin-polarized plasma with $N_{2} \gg N_{1}$ can be reached. We note that the polarization of the EM wave is crucial. In the limit considered here $(\omega \gg \Omega)$, the spin contribution to the ponderomotive force has opposite direction for RCP and LCP waves. Thus, an experiment along these lines must use circular polarized rather than linearly polarized light, as the effect is a factor $\Omega / \omega$ smaller in the latter case. A related use of the ponderomotive force for isotope separation has been suggested in Ref. [20], where the different charge-to-mass ratios of different isotopes was used.

Next, we compare the back-reaction on the EM-pulse, induced by the classical density perturbation $N_{1}$ and its spin-polarized counterpart $N_{2}$. From the classical current $\mathbf{J}=q\left(n_{u} \mathbf{v}_{u}+n_{d} \mathbf{v}_{d}\right)=q N_{1} \mathbf{v}$, we have

$$
J_{ \pm}=q N_{1} v_{ \pm}=i q^{2} N_{1} E_{ \pm} /[m(\omega \pm \Omega)]
$$

with $\mathbf{v}_{u}=\mathbf{v}_{d}$ for RCP and LCP waves. The magnetization current $\mathbf{J}_{\mathrm{M}}=\nabla \times\left(\mathbf{M}_{u}+\right.$ $\left.\mathbf{M}_{d}\right)=(\mu / \hbar)\left[\nabla \times\left(n_{u} \mathbf{S}_{u}+n_{d} \mathbf{S}_{d}\right)\right]$, i.e., $\quad \mathbf{J}_{\mathrm{M} \pm}=$ $\pm\left(k g \mu_{B} / 2 \hbar\right)\left(n_{u} \mathbf{S}_{u \pm}+n_{d} \mathbf{S}_{d \pm}\right)$, together with Eq. (10) and the lowest order expression of $B_{ \pm}$[see Eq. (6)], gives

$$
J_{\mathrm{M} \pm}= \pm 8 i(k \mu / \omega \hbar)^{2}\left[\omega S_{0} /\left(\omega \pm \omega_{g}\right)\right]\left(N_{2} E_{ \pm}\right) .
$$

Now, Eq. (17) can be integrated for $N_{2}$. A Gaussian pulse $|E|=E_{0} \exp \left(-\xi^{2} / L_{p}^{2}\right)$ gives (for $B_{0}, \omega_{g} \rightarrow 0$ )

$$
N_{2}= \pm \frac{\varepsilon_{0} \omega_{p}^{2} k^{2} S_{0}\left|E_{0}\right|^{2} \exp \left(-2 \xi^{2} / L_{p}^{2}\right)}{m^{2} \omega^{3} v_{g}^{2}}\left(1+\frac{v_{g}}{v_{p}}\right) .
$$

Furthermore, for a pulse length much larger than the plasma oscillation wavelength $\lambda_{p}$, we can use the following estimate from Eq. (16) as $\left(B_{0}, \Omega \rightarrow 0\right)$

$$
N_{1} \sim\left[\varepsilon_{0} \omega_{p}^{2}\left|E_{0}\right|^{2} \exp \left(-2 \xi^{2} / L_{p}^{2}\right)\right] /\left(m \omega^{2} v_{g}^{2} L_{p}^{2} k_{p}^{2}\right) .
$$

The density ratio for RCP and LCP waves is then given by

$$
\left|\frac{N_{2}}{N_{1}}\right| \sim\left(\frac{\hbar \omega_{p}}{m c^{2}}\right)\left(k L_{p}\right)^{2}\left(\frac{c}{v_{p}}\right)^{2}\left(\frac{\omega \omega_{p}}{k^{2} v_{g}^{2}}\right)\left(1+\frac{v_{g}}{v_{p}}\right),
$$

where $k_{p} \equiv 1 / \lambda_{p}$. The ratio of the two currents for RCP and LCP waves is then given by $\Gamma \equiv\left|J_{\mathrm{M} \pm} / J_{ \pm}\right| \approx$ $\left(\hbar \omega / m v_{p}^{2}\right)\left|N_{2} / N_{1}\right|$, i.e.

$$
\Gamma \sim\left(\hbar \omega_{p} / m c^{2}\right)^{2}\left(k L_{p}\right)^{2}\left(c^{2} / v_{p} v_{g}\right)^{2}\left(1+v_{g} / v_{p}\right) .
$$

Thus, for $v_{g}, v_{p} \sim c$, we have

$$
\Gamma \sim\left(\hbar \omega_{p} / m c^{2}\right)^{2}\left(k L_{p}\right)^{2} .
$$

Solving Eqs. (16) and (17), we in Fig. 2 compare the two current profiles for an XFEL with $\lambda=1 \mathrm{~nm}$, a pulse length $L_{p}=30 \mu \mathrm{m}$, and a metallic plasma density, giving $\omega_{p} / 2 \pi=10^{16} \mathrm{~s}^{-1}$. Our estimate (24) is then verified, and it is found that the central value of the current ratio is $\Gamma \approx 3$. These parameters are relevant for the XFEL at DESY [21]. In fact, the shortest wavelength generated by this facility is $\lambda=0.1 \mathrm{~nm}$, making the quantum mechanical back-reaction much larger than the classical response $[\Gamma \sim 200$ according to Eq. (24)].

In the present Letter, we have generalized the classical expression for the ponderomotive force in a magnetized plasma to include the effect of the electron spin. Our main result, Eq. (13), applies for arbitrary electromagnetic waves propagating along an external magnetic field. One of the main features of the spin-ponderomotive force is that it can induce a strong spin-polarization in a 


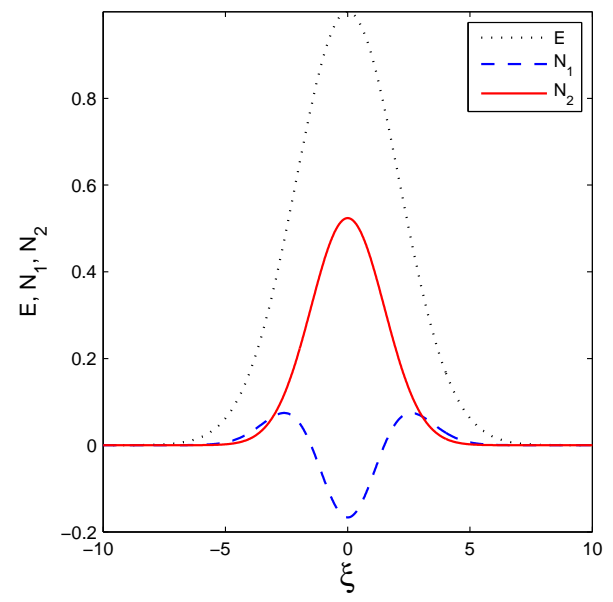

FIG. 1. The normalized classical density perturbation $N_{1}$ and the spin-induced density difference $N_{2}$ together with a Gaussian EM-pulse, $|E|=\left|E_{0}\right| \exp \left(-\xi^{2} / L_{p}^{2}\right)$, as calculated from Eqs. (16) and (17). The parameters correspond to an unmagnetized plasma with $\omega_{p} / 2 \pi=10^{16} \mathrm{~s}^{-1}, \lambda=80 \mathrm{~nm}$, and $L_{p}=15 \mu \mathrm{m}$ (a.u.).

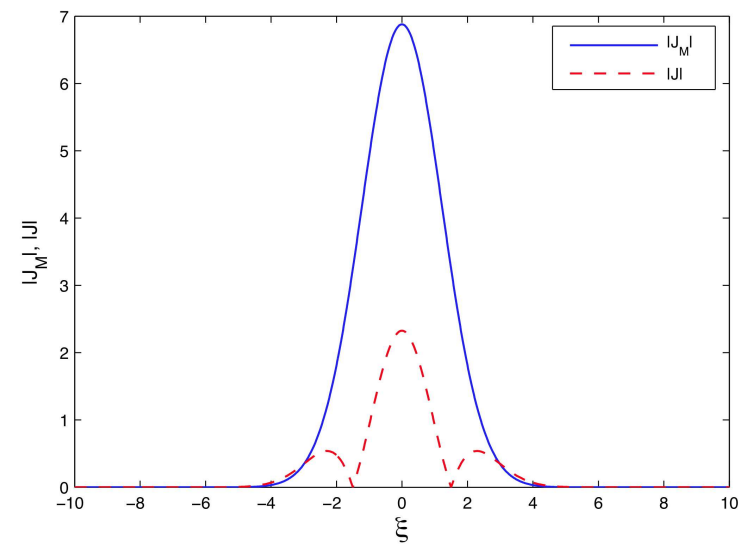

FIG. 2. The profiles of the normalized spin-induced current density $J_{M}$ and the classical current density $J$ for a Gaussian EM-pulse, as obtained from Eqs. (18) and (19). The parameters correspond to an unmagnetized plasma with $\omega_{p} / 2 \pi=10^{16} \mathrm{~s}^{-1}, \lambda=1 \mathrm{~nm}$, and $L_{p}=30 \mu \mathrm{m}$ (a.u.).

plasma, even if the initial up- and down- states of electrons are equally populated. An example with an EMpulse in the UV-regime is given in Fig. 1. Furthermore, even in an unmagnetized plasma, the nonlinear backreaction from the spin-induced current can be larger than the classical back-reaction, provided the EM-pulse has a sufficiently short-wavelength. It should be stressed that we have here only compared the spin-polarized current response with the classical current density, and that relativistic nonlinearities (see e.g. 7]) that has been omitted here may play a role for the full dynamic evolution. An example with an XFEL is given in Fig. 2. Finally, we want to point out that the possibilities of nonlinear spin effects is still a relatively unexplored area, and generalizations, such as arbitrary directions of propagation, are likely to lead to new and interesting discoveries.

A.P.M. acknowledges support from the Kempe Foundations and thanks B. Eliasson for help regarding numerical simulations. This research is supported by the European Research Council and the Swedish Research Council under Contracts \# 204059-QPQV and 2007-4422.

* mattias.marklund@physics.umu.se

[1] S. A. Wolf et al., Science, 294, 1488 (2001)

[2] D. Kremp, M. Schlanges and W. -D. Kraft, Quantum Statistics of Nonideal Plasmas, (Springer, 2005).

[3] M. Marklund and G. Brodin, Phys. Rev. Lett. 98, 025001 (2007)

[4] G. Brodin, M. Marklund, and G. Manfredi, Phys. Rev. Lett. 100, 175001 (2008)

[5] L. M. Gorbunov and V. I. Kirsanov, Zh. Eksp. Teor. Fiz. 93, 509 (1987) [Sov. J. Plasma Phys. 93, 290 (1987)].

[6] R. Bingham, Nature 445, 721 (2007).

[7] L. Bergé, Phys. Reports 303259 (1998); P. K. Shukla, N. N. Rao, M. Y. Yu and N. L. Tsintsadze, Phys. Reports, 138, 1 (1986).

[8] W. L. Kruer, The Physics of Laser Plasma Interactions (Westview Press, Boulder, 2003).

[9] P. K. Shukla and B. Eliasson, Phys. Rev. Lett. 99, 096401 (2007); P. K. Shukla, N. Shukla and L. Stenflo, J. Plasma Phys. 76, 25, (2010).

[10] M. Idrish Miah, Sci. Technol. Adv. Mater. 9, 035014 (2008).

[11] S.J. Chen and D. H. E. Dubin, Phys. Fluids B, 5, 691, (1993)

[12] Effects like the Fermi pressure or Bohm-de Broglie dispersion [13], relevant for low-temperature and/or high density plasmas, is straightforward to add. However, such phenomena only occur when density perturbations are involved, and hence the ponderomotive force, resulting from quadratic nonlinearities in the transverse variables, is not affected within a fluid theory.

[13] P. K. Shukla and B. Eliasson, Phys. Usp. 53, 51 (2010).

[14] V.I. Karpman and H. Washimi, J. Plasma Phys. 18, 173 (1977).

[15] D. Bauer, P. Mulser and W. H. Steeb, Phys. Rev. Lett. 75, 4622 (1995).

[16] I. Y. Dodin and N. J. Fisch, Phys. Rev. E 77, 036402 (2008).

[17] J. R. Cary and A. N. Kaufman, Phys. Fluids, 24, 1238 (1981); M. Kono and H. Sanuki, J. Plasma Phys. 38, 43 (1987).

[18] P. K. Shukla, G. Brodin, M. Marklund and L.Stenflo, Phys. Lett. A 373, 3165, (2009).

[19] The Fermi pressure and the Bohm-de Broglie potential has been shown to influence the longitudinal dynamics excited by the ponderomotive force [18]. However, for the case when $v_{g} \gg v_{F}$ (where $v_{F}$ is the Fermi velocity), the physics on length scales much longer than the Compton wavelength, which here is our concern, is unaffected.

[20] E. S. Weibel, Phys. Rev. Lett. 44, 377 (1980).

[21] See, e.g., http://xfel.desy.de/ 